\title{
Facebook as an Instrument of Political Participation in the Youth of Pakistan
}

\author{
Komal Ahmed \\ Assistant Professor \\ Department of Media Studies \\ Kinnaird College for Women, Lahore - Pakistan \\ Komal.Ahmed@Kinnaird.Edu.Pk \\ $\&$ \\ Dr. Maryam Azam \\ Assistant Professor \\ Department of International Relations \\ Lahore College for Women University \\ Maryam.Azam@Ymail.Com \\ $\&$ \\ Dr. Ayesha Siddiqua \\ Lecturer \\ Department of Mass Communication \\ National University of Modern Languages, Islamabad \\ Asiddiqua@Numl.Edu.Pk
}

\begin{abstract}
The inclusion of social media in politics has brought major transformations in the political culture and narrative building. In turn, it has massive impact on shaping and reshaping the public opinion. In addition, it also influences the perception of policy makers and leaders. In this context, this paper entails that how Facebook played a significant role in bringing youth as an active agent in the politics of Pakistan. The results identify that use of Facebook played a significant role in increasing youth participation and their interest in politics.
\end{abstract}

\section{INTRODUCTION}

Facebook has evolved in to a source of engagement and connectivity for the masses. The marketing strategies of Facebook ensure high engagement of social organizations which in turn compel people to talk about sensitive topics and raise their voices. Due to technological advancement people can now access 
internet through their mobile phones, tablets, iPods etc. Youth today gather information about political activities from social media and start giving their responses in return. In this context, Facebook is one of the largest mediums within Social Networking Sites (SNS) worldwide. People mostly participate in politics from their face book account through following possible ways:

- People can post ideas, stories, opinions etc.

- People like and share posts which can reflect their interests, support or endorsement towards a particular theme

- People can participate in online debates

- Users upload photos, videos and send messages

- Facebook users can make and join Facebook pages and groups

The above options illustrate that Facebook users have diverse options for online interaction. Moreover, they can further increase the interaction and audiences for their content by making it public. In this context, it is being observed that Facebook has become a popular source of connectivity, publicity and interaction used by the political parties, leaders, politicians, and by the governments to seek connectivity and support. In turn, they are being followed by the youth. Facebook political participation was first started primarily in the 2008 U.S elections. Consequently, this culture of political participation through smart technology gained popularity across the globe. The use of social media in Arab spring further expanded its involvement in politics. In Pakistan, primarily the election campaign of the 2013 general election was the first major political activity in which social media was been used as a medium of connectivity and political campaign. In this context, youth population in particular participated in this new trend of online participation.

Velasquez and LaRose (2014) described that there are a lot of studies done on youngster's political involvement and social media networks but there is less study done on youth social and political mobilization in a form of group because when youngsters use social media as a platform to tell their thoughts then it is done together. Unlike, that an individual mobilizes and take part in political activities. Youngsters get together and then raise their voices in a political setting. It explains the association between youth and online shared political activism. The researcher tells that youngsters are interdependent on each other in order to create mobilization. Similarly, Ward (2013) examined that authenticity plays a main role in online political campaigning on social networking websites. Inaccurate and false information is frequently uploaded online which creates chaos and problems. Political campaigns on social networks were majorly started in 2008 presidential elections in United States since then it spreads across the world and now it is important part of every election campaign. Validity is essential so that every information and data that is provided is accurate and individuals take full benefit from it.

Since a decade, the use of social media has been extensively increased in Pakistan. In 2019, there were 31,467,000 Facebook users in Pakistan which accounts for 14.8 percent of the total 
population. Interestingly, the youth population aged from 18 to 24 years is the largest group who use Facebook (www.napolecat.com, 2019). There is a significant correlation between use of Facebook and political participation. Therefore, online youth participation influences the offline youth participation. The study aims to analyze the impact of Facebook on youth in terms of political participation particularly during the election campaign of the general elections in 2013. As seen in the 2013 general elections in Pakistan, Facebook increased youth mobilization because they were following Facebook political campaigns to get news about elections. For this reason, each party and their leaders had official pages and accounts on which they uploaded statuses (Siddiqui, 2013). There was a massive turnout of youth activism and mobilization in the country, one major cause was Facebook as election campaign was at its glance on it. Nearly every aspect of election campaign included social media in one way or another. Social media helped electoral candidates to communicate their thoughts, program, and messages to the community easily. During the 2013 elections, political parties including Pakistan Tehrek Insaf (PTI), Pakistan Muslim League-Nawaz (PML-N), Pakistan People's Party (PPP), Jamiat Ulema Islam (JUI), Jamait -e- Islami (JI), Mutehida Qoumi Movement (MQM), Awami National Party (ANP) made social media cells in order to run political campaigns. In this entire process, youth were most enthusiastic and determined about the elections. Social media introduced a new trend in the politics of Pakistan. About 40 million young Pakistanis voted for the first time. The 2013 general elections marked a 60 percent of massive voter turnout because of the election campaigns in media and especially in social media (Siddiqui, 2013). Furthermore, Adeney (2013) has examined that for the first time in Pakistan, there was a smooth civilian transition of the government through the conduct of the general elections after five years. The election campaign of 2013 on Facebook was massive. Pakistan Tehreek-e-Insaf was pioneer in starting the campaign on social media which was then followed by other political parties. Awami National Party (ANP), Pakistan People Party (PPP), and mainly Muttahida Qaumi Movement (MQM) slow down their campaigns and used social media after threats received by terrorist. Facebook campaign increased interest of people to vote for their right especially in major urban centers of Pakistan. Similarly, Ahmad and Sheikh (2013) endorsed that youth became politically mobilized due to social media. It brought a major change in traditional politics patterns of Pakistan. In the 2013 elections, youth political engagement increased because they freely took part in it without any gate keeping method and gave their opinions, launched campaign for their favorite political party or leader by updating statuses, uploading cover photos and profile pictures. Another study related to the youth participation in the 2013 general elections by Rosheen Kabraji (2013) examined that out of total population in Pakistan over 66\% are under the age of thirty. Therefore, youth is in large number and can affect the political situation.

\section{THEORETICAL FRAMEWORK}

\section{Agenda Setting Theory}

The agenda setting theory was presented by McCombs and Shaws (1993) in 1972 and it explicates that media has a very strong influence on its audience. It states that media focuses on 
particular targeted issues which influences the audience by prioritizing the issue or a particular activity through media strategies. The theory strongly focuses on the gatekeeping process which is the process of filtering information that the media thinks is more relevant due to different reasons (Zain, 2014).

McCombs and Shaw observed the presidential campaigns during the elections of 1968 , 1972 and 1976 and found that mass media has a great influence on the behavioral patterns of the audience. It affects how people behave after they are informed about a particular issue through mass media. According to the theory, media has the power to set public agenda most significantly in matters that include attitudes and opinions. It has access in influencing and contributing the change in perceptions, priorities, focus and values of the audience. With such a high tendency of stimulating, the audiences form their opinion and focuses on issues that are considered to be important, even for them (Zain, 2014). Therefore, agenda setting theory plays a vital role in shaping and reshaping opinions and perceptions.

When an issue gains importance in the media then it affects public understanding as they try to talk, discuss and explore about the concerned topic. Agenda setting is successful only when topic is relevant for the public otherwise public will not consider it as important (Zain, 2014). Agenda setting theory relates the public opinion formation to awareness framed by the media. The print and television media in Pakistan mostly used to create propaganda and false news, but with the invention of social media networks nothing can be hidden. It fills the gap because there is no gate keeping and filtering in the content. Thus, social media and especially Facebook gives information faster as compared to traditional media. Its activities and information about political events and situations mobilizes youth to take interest and part in it. Facebook has made youth conscious about the political scenario in Pakistan. In this perspective, following quantitative data entails the link between the use of Facebook and youth participation in Pakistan.

\section{Research Questions:}

- What is the popularity of Facebook among youth?

- To what extent Facebook is used by youngsters for political participation?

- Does Facebook create impact on youth regarding political participation?

This study used survey research as the method to draw out inferences. A closed ended questionnaire has been constructed. The survey method is used to study and examine the behavior of individuals. The sample size is 250 respondents. The selected age group is 18-25. For data analysis, SPSS and Minitab software is used in this research. In this study, independent variable is the political participation on Facebook and dependent variable is youth.

\section{Hypothesis No. I}


$\mathbf{H}_{1:}$ Facebook is used for political participation among youth

$\mathbf{H}_{\mathbf{o}}$ : Facebook is not used for political participation among youth

One proportion test was applied in Mini Tab statistical software

Confidence Level: $95 \%$

Level of Significance $=0.5$

Test and CI for One Proportion

\begin{tabular}{|c|c|c|c|c|c|}
\hline \multicolumn{6}{|c|}{ Test of $p=0.5$ vs $p>0.5$} \\
\hline \multicolumn{6}{|c|}{ Exact } \\
\hline Sample & $\mathrm{X}$ & $\mathrm{N}$ & Sample p 95\% & Lower Bound & P-Value \\
\hline 1 & 165 & 250 & 0.660000 & 0.607477 & 0.000 \\
\hline
\end{tabular}

Interpretation: The proportion test revealed that 165 respondents answered as yes that they use Facebook for political participation. As the $\mathrm{P}$ value for the hypothesis is 0.000 which specifies that the result is highly significant. Null hypothesis is proven wrong; therefore, the result concluded that youth is using Facebook for participating in politics.

\section{Hypothesis No. 2:}

H1: Facebook has an impact on youth regarding political participation

$\mathbf{H}_{\text {o: }}$ Facebook has no impact on youth regarding political participation

One proportion test was applied in Mini Tab statistical software

Confidence Level: $95 \%$ 
Level of Significance $=0.5$

\section{Test and CI for One Proportion}

\begin{tabular}{|l|l|l|l|l|l|}
\hline \multicolumn{5}{|c|}{ Test of $p=0.5$ vs $p>0.5$} \\
\hline \multicolumn{5}{|l|}{ Exact } \\
\hline 1
\end{tabular}

Interpretation: The proportion test revealed that 182 respondents answered as yes that Facebook has an impact on youth regarding political participation. As the $\mathrm{P}$ value for the hypothesis is 0.000 which specifies that the result is highly significant. Null hypothesis is proven wrong; therefore, the result concluded that Facebook has an impact on youth for participation in politics.

\section{DISCUSSION AND ANALYSIS:}

Q.1: Age of respondents: 
Figure 1.1 shows the age of respondents

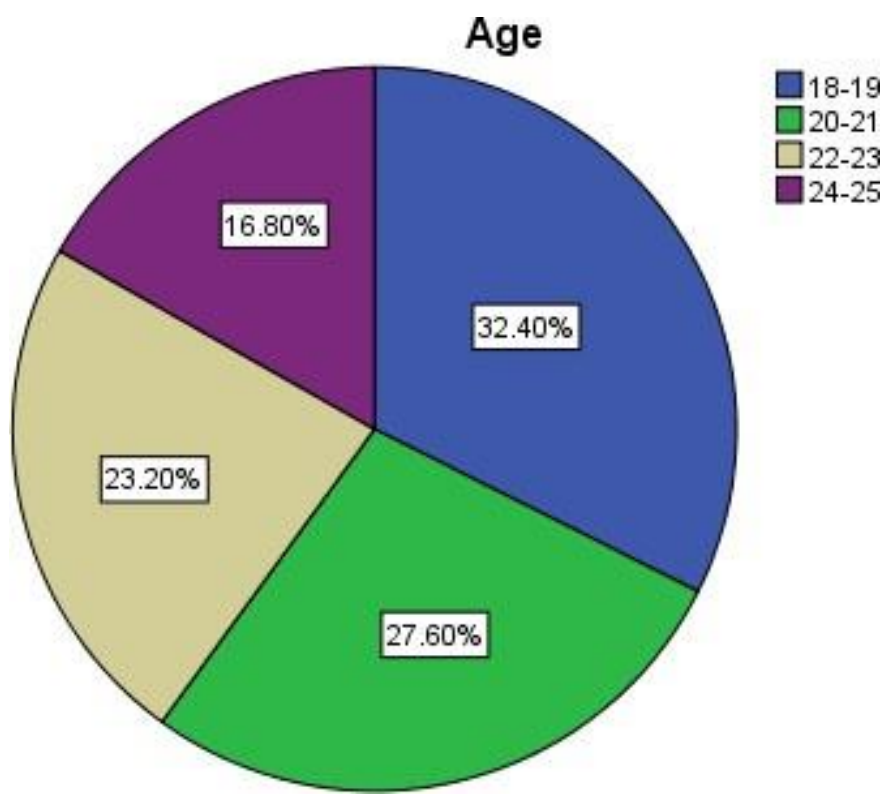

Table 1.1 shows the age of respondents

\begin{tabular}{|r|r|r|r|r|}
\hline & Frequency & Percent & Valid Percent & \multicolumn{2}{c|}{$\begin{array}{c}\text { Cumulative } \\
\text { Percent }\end{array}$} \\
\hline $18-19$ & 81 & 32.4 & 32.4 & 32.4 \\
$20-21$ & 69 & 27.6 & 27.6 & 60.0 \\
$22-23$ & 58 & 23.2 & 23.2 & 83.2 \\
$24-25$ & 42 & 16.8 & 16.8 & 100.0 \\
Total & 250 & 100.0 & 100.0 & \\
\hline
\end{tabular}

Table 1.1 shows the sampling age group which is $18-25.32 .4 \%$ are between the ages of $18-19$, $27.6 \%$ are among 20-21 age set, $23.3 \%$ are between ages $22-23$ and 16.8\% are from age of 24-25.

Q 2: How much time do you spend on Facebook daily? 
Figure 1.2 shows the time spend on Facebook daily how much time do you spend on facebook daily?

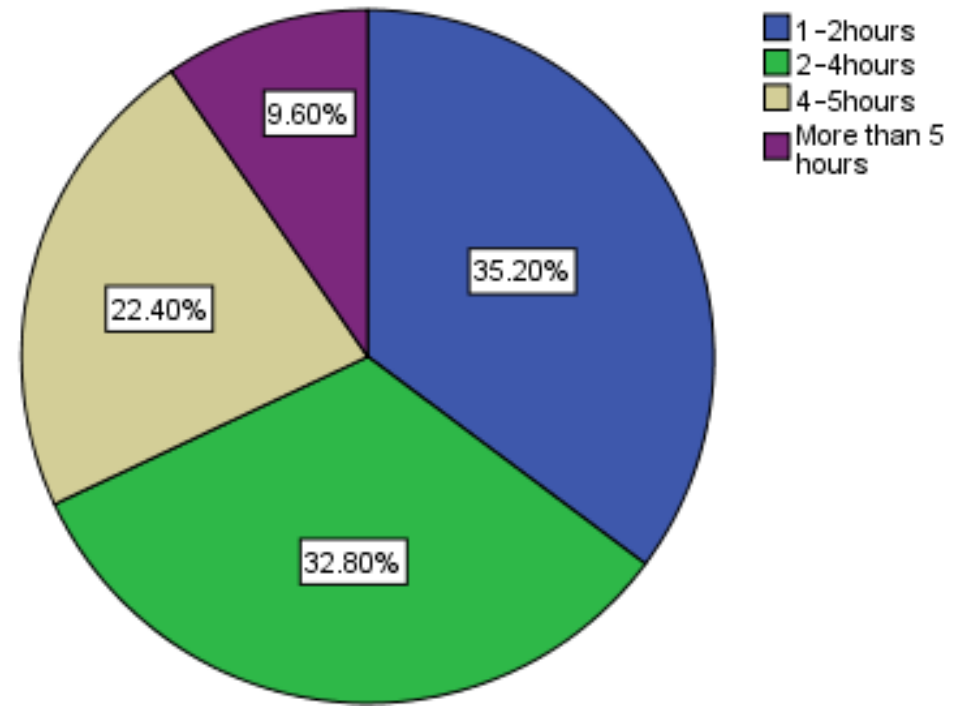

Table 1.2 shows the time spend on Facebook daily

\begin{tabular}{|l|r|r|r|r|}
\hline & Frequency & Percent & Valid Percent & $\begin{array}{c}\text { Cumulative } \\
\text { Percent }\end{array}$ \\
\hline 1-2hours & 88 & 35.2 & 35.2 & 35.2 \\
2-4hours & 82 & 32.8 & 32.8 & 68.0 \\
4-5hours & 56 & 22.4 & 22.4 & 90.4 \\
More than 5 hours & 24 & 9.6 & 9.6 & 100.0 \\
Total & 250 & 100.0 & 100.0 & \\
\hline
\end{tabular}

Table 1.2 shows the time spend on Facebook daily. 35.2\% of respondents spends 1-2hours, $32.8 \%$ spends $2-4$ hours while $22.4 \%$ spends $4-5$ hours and $9.6 \%$ spends more than 5 hours on Facebook daily.

Q3: Do Facebook encourage you to participate in politics? 
Figure 1.3 shows Facebook encourage towards political participation

\section{Do facebook encourage you to participate in politics?}

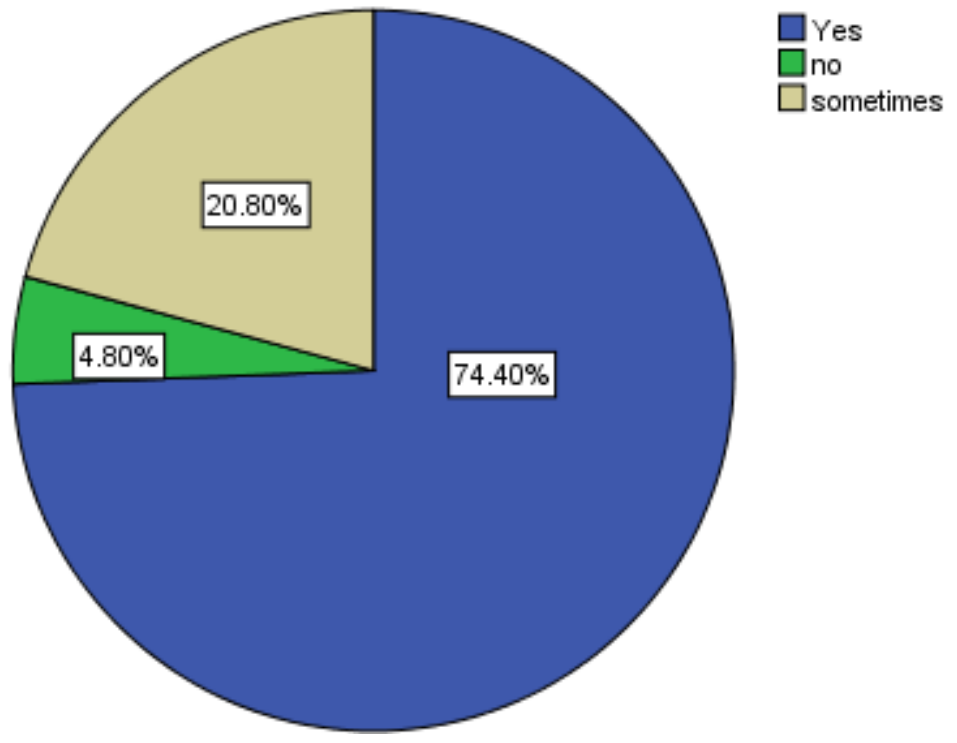

Table 1.3 shows Facebook encourage towards political participation

\begin{tabular}{|l|r|r|r|r|}
\hline & Frequency & Percent & Valid Percent & $\begin{array}{c}\text { Cumulative } \\
\text { Percent }\end{array}$ \\
\hline Yes & 186 & 74.4 & 74.4 & 74.4 \\
No & 12 & 4.8 & 4.8 & 79.2 \\
Sometimes & 52 & 20.8 & 20.8 & 100.0 \\
Never & 0 & 0.0 & 0.0 & 0.0 \\
Total & 250 & 100.0 & 100.0 & \\
\hline
\end{tabular}

Table 1.3 shows the respondent opinion that Facebook encourages youth to participate in politics to which $74.8 \%$ answered yes, $4.4 \%$ responded no, $20.8 \%$ replied sometimes and $0 \%$ responded as never.

Q.4: Do you think that Facebook is popular among youth for political participation? 
Figure 1.4 shows Facebook is popular among youth for political participation

\title{
Do you think that Facebook is popular among youth for political participation?
}

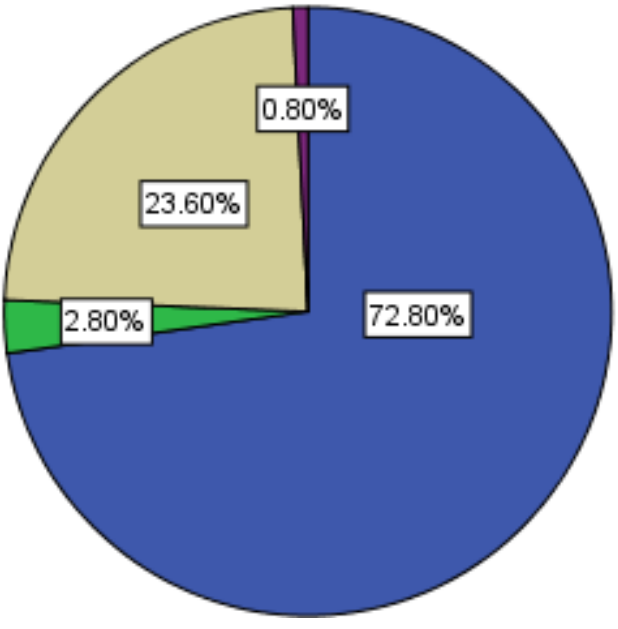

\author{
$\square$ yes \\ 口no \\ $\square$ sometimes \\ $\square$ never
}

Table 1.4 shows Facebook is popular among youth for political participation

\begin{tabular}{|l|r|r|r|r|}
\hline & Frequency & Percent & Valid Percent & $\begin{array}{c}\text { Cumulative } \\
\text { Percent }\end{array}$ \\
\hline Yes & 182 & 72.8 & 72.8 & 72.8 \\
No & 7 & 2.8 & 2.8 & 75.6 \\
Sometimes & 59 & 23.6 & 23.6 & 99.2 \\
Never & 2 & .8 & .8 & 100.0 \\
Total & 250 & 100.0 & 100.0 & \\
\hline
\end{tabular}

Table 5.5 shows responded opinion that Facebook is popular among youth for political participation. $72.8 \%$ replied yes, $2.8 \%$ replied No, $23.6 \%$ answered sometimes while $8 \%$ replied Never.

Q 5: Is Facebook main source of political news for you? 
Figure 1.5 shows Facebook as a source to get political news

\section{Is Facebook main source of political news for you?}
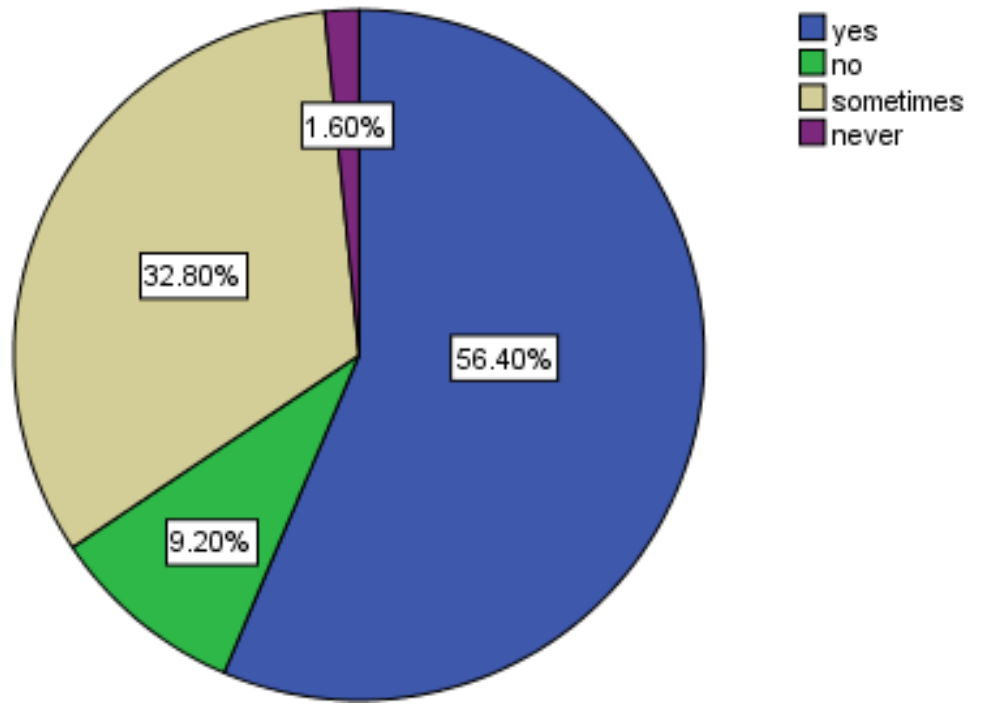

Table 1.5 shows Facebook as a source to get political news

\begin{tabular}{|l|r|r|r|r|}
\hline & Frequency & Percent & Valid Percent & \multicolumn{2}{c|}{$\begin{array}{c}\text { Cumulative } \\
\text { Percent }\end{array}$} \\
\hline Yes & 141 & 56.4 & 56.4 & 56.4 \\
No & 23 & 9.2 & 9.2 & 65.6 \\
Sometimes & 82 & 32.8 & 32.8 & 98.4 \\
Never & 4 & 1.6 & 1.6 & 100.0 \\
Total & 250 & 100.0 & 100.0 & \\
\hline
\end{tabular}

Table 1.5 shows that Facebook is the main source of political news for youth. $56.4 \%$ responded as yes, $9.2 \%$ replied No, while $32.8 \%$ answered Sometimes and 1. 6\% responded as never.

Q 6: Is Facebook the best platform to express your thoughts and opinions? 
Figure 1.6 shows Facebook as a platform to express thoughts and opinions

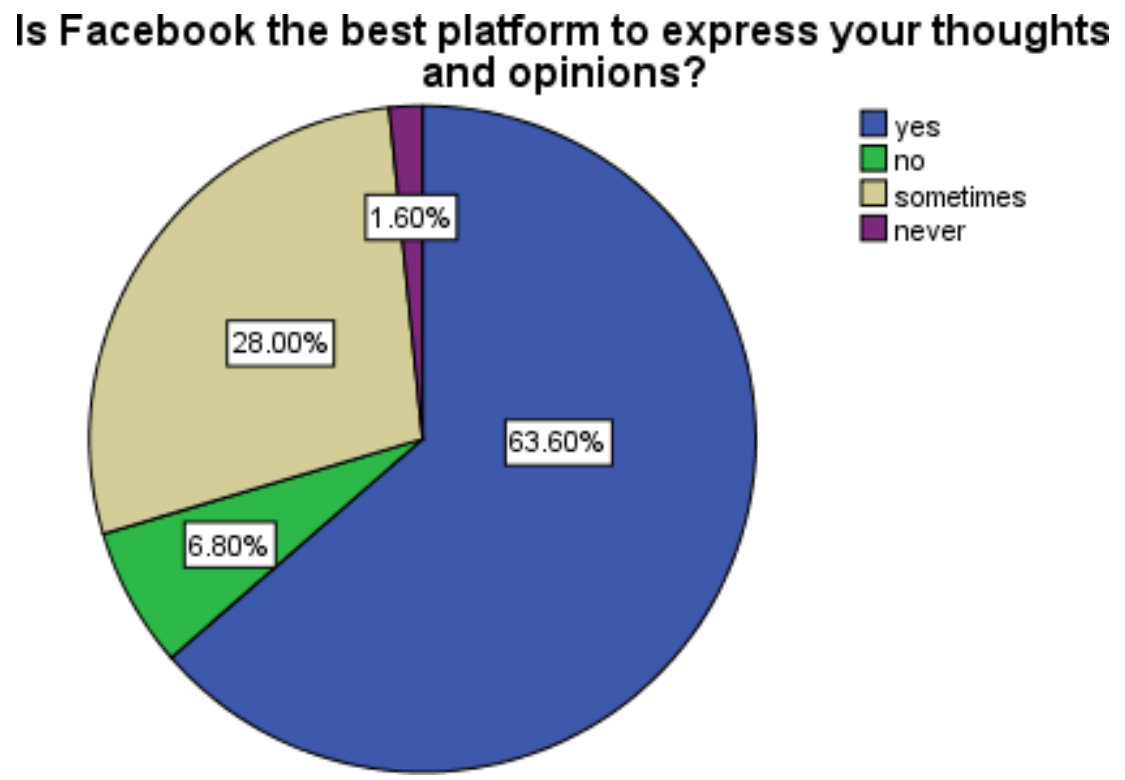

Table 1.6 shows Facebook as a platform to express thoughts and opinions

\begin{tabular}{|l|r|r|r|r|}
\hline & Frequency & Percent & Valid Percent & \multicolumn{2}{|c|}{$\begin{array}{c}\text { Cumulative } \\
\text { Percent }\end{array}$} \\
\hline Yes & 159 & 63.6 & 63.6 & 63.6 \\
No & 17 & 6.8 & 6.8 & 70.4 \\
Sometimes & 70 & 28.0 & 28.0 & 98.4 \\
Never & 4 & 1.6 & 1.6 & 100.0 \\
Total & 250 & 100.0 & 100.0 & \\
\hline
\end{tabular}

Table 1.6 shows that Is Facebook the best platform to express your thoughts and opinions to which $63.6 \%$ answered yes, $6.8 \%$ said No, and $28.0 \%$ replied sometimes while $1.6 \%$ responded Never.

Q 7: Do you think Facebook impacts on political participation of youth? 
Figure 1.7 shows Facebook impacts on political participation

\section{Do you think Facebook impacts on political participation of youth?}
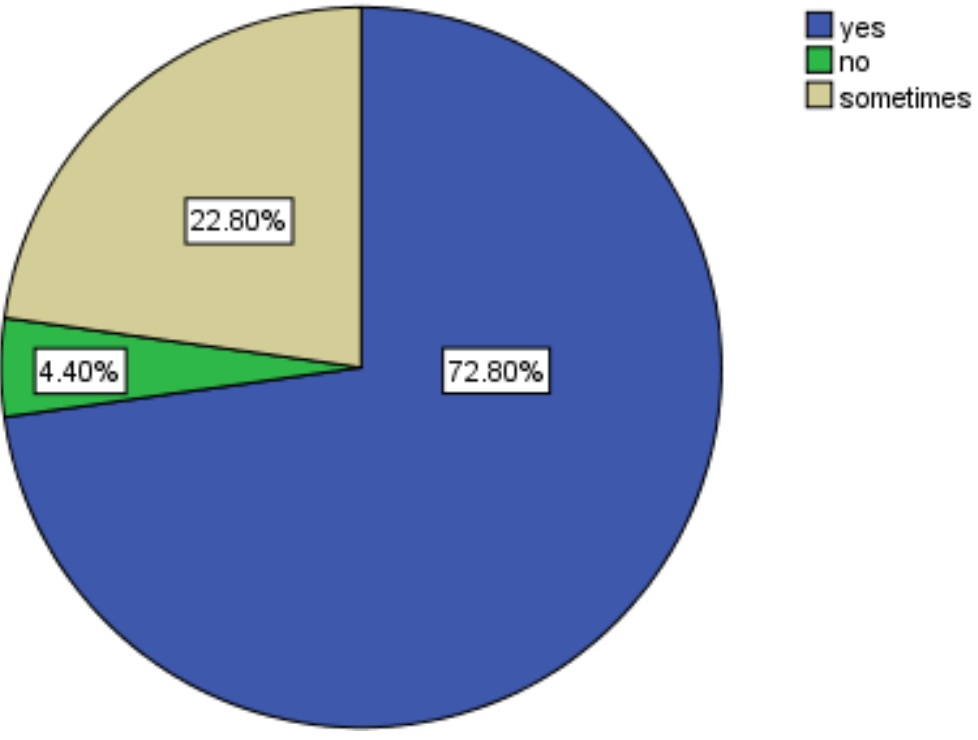

Table 1.7 shows Facebook impacts on political participation

\begin{tabular}{|l|r|r|r|r|}
\hline & Frequency & Percent & Valid Percent & $\begin{array}{c}\text { Cumulative } \\
\text { Percent }\end{array}$ \\
\hline Yes & 182 & 72.8 & 72.8 & 72.8 \\
No & 11 & 4.4 & 4.4 & 77.2 \\
Sometimes & 57 & 22.8 & 22.8 & 100.0 \\
Never & 0 & .0 & .0 & 0.0 \\
Total & 250 & 100.0 & 100.0 & \\
& & & & \\
\hline
\end{tabular}

Table 1.7 shows that Facebook impacts on political participation of youth. $72.8 \%$ of the respondents answered yes, $4.4 \%$ responded no, $22.8 \%$ answered Sometimes while 0\% replied Never.

Q 8: Do you think youth spent most of their time on Facebook? 
Figure 1.8 shows youth spending more time at Facebook

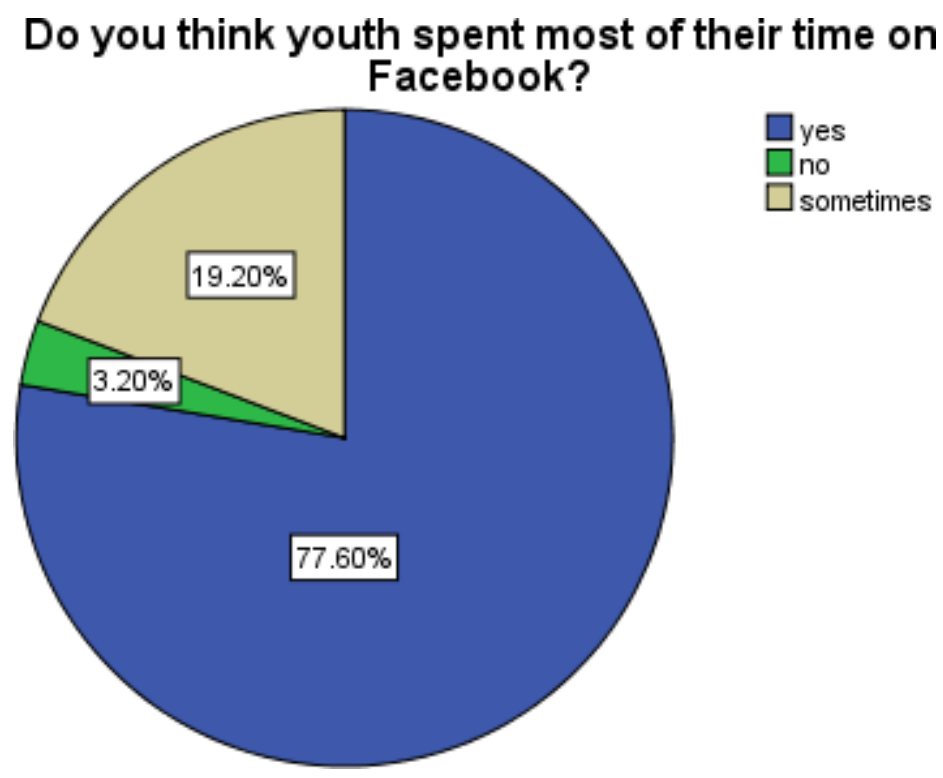

Table 1.8 shows youth spent most of their time on Facebook

\begin{tabular}{|l|r|r|r|r|}
\hline & Frequency & Percent & Valid Percent & $\begin{array}{c}\text { Cumulative } \\
\text { Percent }\end{array}$ \\
\hline Yes & 194 & 77.6 & 77.6 & 77.6 \\
No & 8 & 3.2 & 3.2 & 80.8 \\
Sometimes & 48 & 19.2 & 19.2 & 100.0 \\
Never & 0 & .0 & .0 & 0.0 \\
Total & 250 & 100.0 & 100.0 & \\
\hline
\end{tabular}

Table 1.8 shows that youth spend most of their time on Facebook, $77.6 \%$ said yes that youth spends most of their time Facebook. However, 3.2\% responded as no that they do not spend most of their time on Facebook. Significantly 19.2\% replies that they sometime spend their most of time on Facebook whereas $0 \%$ replied with Never.

Q 9: Do you think youth opinion about political affairs is influenced by Facebook activities? 
Figure 1.9 shows opinions about political affairs influenced by Facebook

\section{Do you think youth opinion about political affairs is influenced by Facebook activities?}
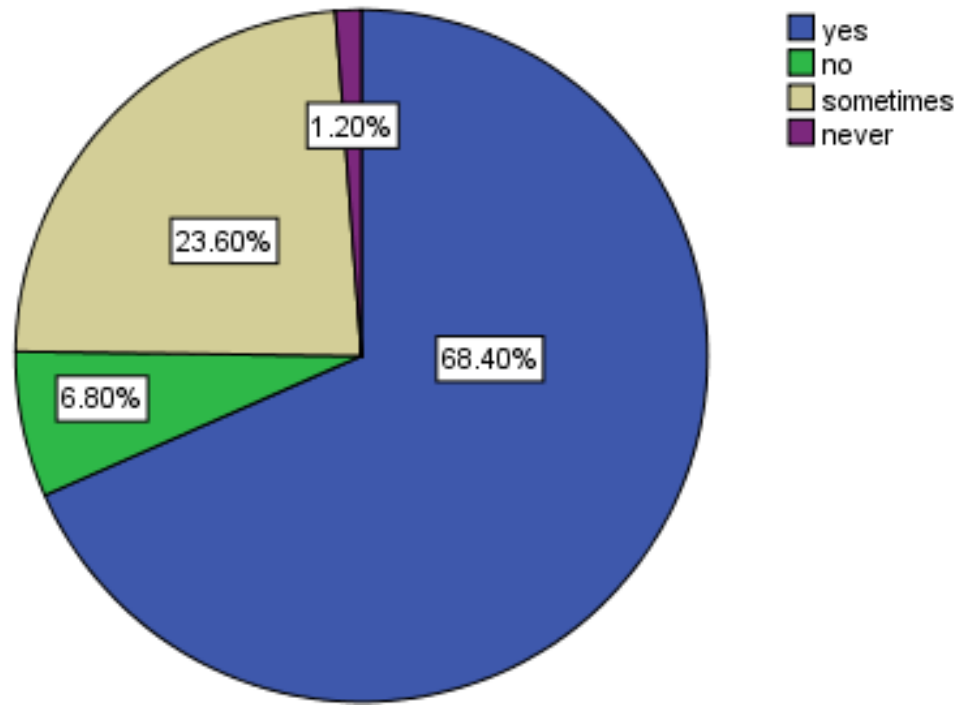

Table 1.9 shows opinions about political affairs influenced by Facebook

\begin{tabular}{|l|r|r|r|r|}
\hline & Frequency & Percent & Valid Percent & $\begin{array}{c}\text { Cumulative } \\
\text { Percent }\end{array}$ \\
\hline Yes & 171 & 68.4 & 68.4 & 68.4 \\
No & 17 & 6.8 & 6.8 & 75.2 \\
Sometimes & 59 & 23.6 & 23.6 & 98.8 \\
Never & 3 & 1.2 & 1.2 & 100.0 \\
Total & 250 & 100.0 & 100.0 & \\
\hline
\end{tabular}

Table 1.9 shows that youth opinion about political affairs is influenced by Facebook activities, $68.4 \%$ responded as yes, $6.8 \%$ replied as no. However, $23.6 \%$ answered sometimes and 1.2\% responded Never.

Q10: Do you believe that Facebook can be used as a political tool? 
Figure 1.10 shows Facebook as a political tool

\section{Do you believe that Facebook can be used as a political tool?}
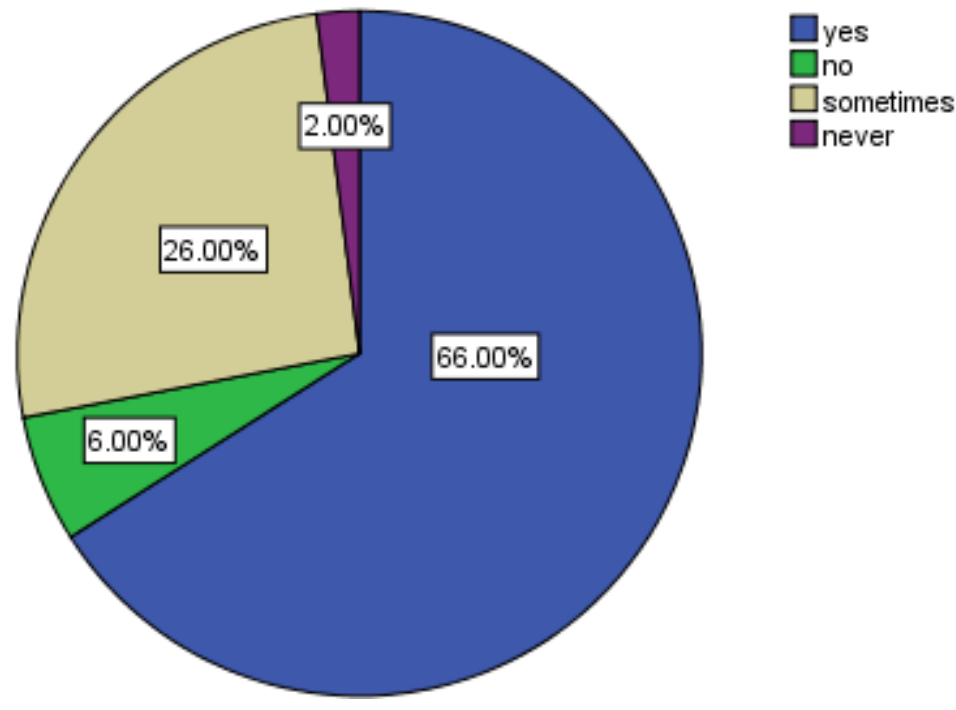

Table 1.10 shows Facebook as a political tool

\begin{tabular}{|ll|r|r|r|r|}
\hline & Frequency & Percent & Valid Percent & $\begin{array}{c}\text { Cumulative } \\
\text { Percent }\end{array}$ \\
\hline \multirow{4}{*}{ Valid } & & & & 66.0 \\
& Yes & 165 & 66.0 & 66.0 & 72.0 \\
& So & 15 & 6.0 & 6.0 & 98.0 \\
& Sometimes & 65 & 26.0 & 26.0 & 100.0 \\
& Nover & 5 & 2.0 & 2.0 & \\
\hline
\end{tabular}

Table 1.10 shows Facebook as a political tool to which $66 \%$ answered as yes that it can be used as a political tool whereas $6 \%$ responded as no that it cannot be used as a political tool. $26 \%$ answered as sometimes it can be used as a political tool however, $2 \%$ responded never.

Q11: Do you think Facebook played a key role to mobilize youth to participate in politics? 
Figure 1.11 shows Facebook as a key role to mobilize youth towards political participation

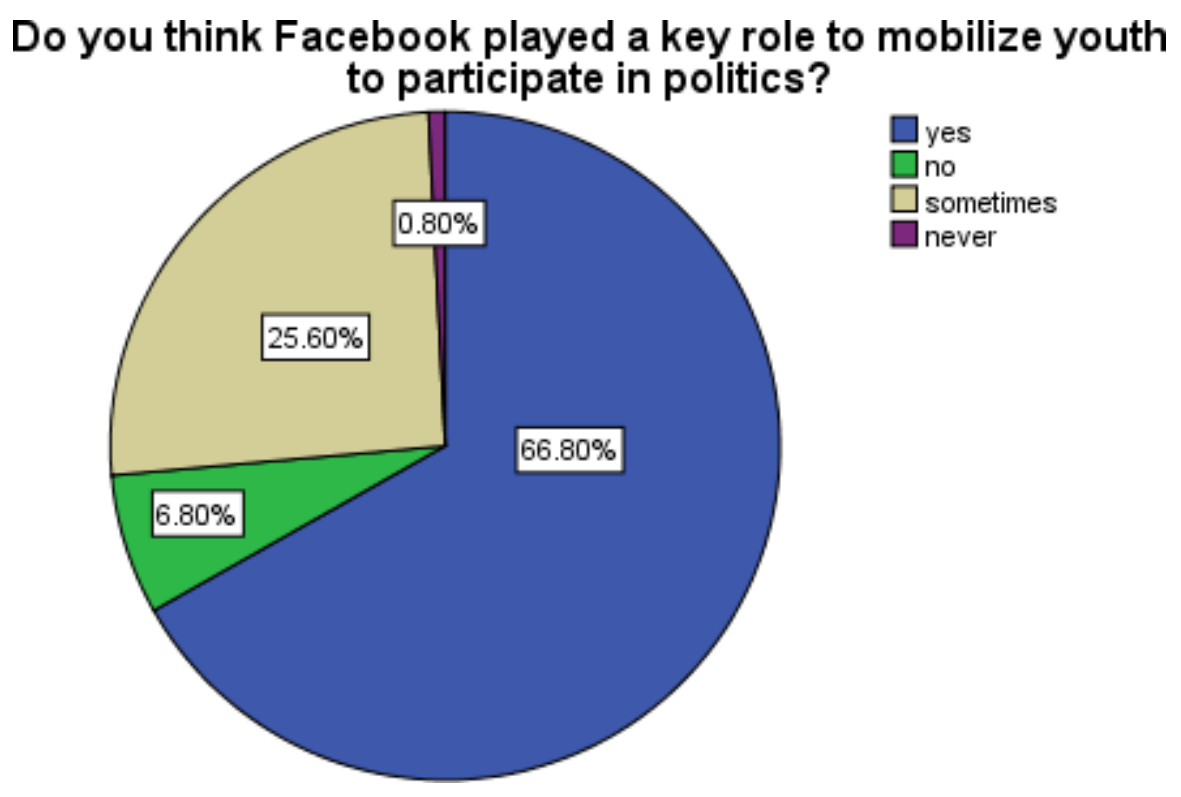

Table 1.11 shows Facebook as a key role to mobilize youth towards political participation

\begin{tabular}{|l|r|r|r|r|}
\hline & Frequency & Percent & Valid Percent & $\begin{array}{c}\text { Cumulative } \\
\text { Percent }\end{array}$ \\
\hline Yes & 167 & 66.8 & 66.8 & 66.8 \\
No & 17 & 6.8 & 6.8 & 73.6 \\
Sometimes & 64 & 25.6 & 25.6 & 99.2 \\
Never & 2 & .8 & .8 & 100.0 \\
Total & 250 & 100.0 & 100.0 & \\
\hline
\end{tabular}

Table 1.11 shows that Facebook played a key role to mobilize youth to participate in politics. $66.8 \%$ responded as yes, $6.8 \%$ answered as no, $25.6 \%$ responded sometimes while $8 \%$ answered as never.

Q.12: Do you think Facebook help you to get updates about political scenario? 
Figure 1.12 shows Facebook helps youth to get update regarding politics

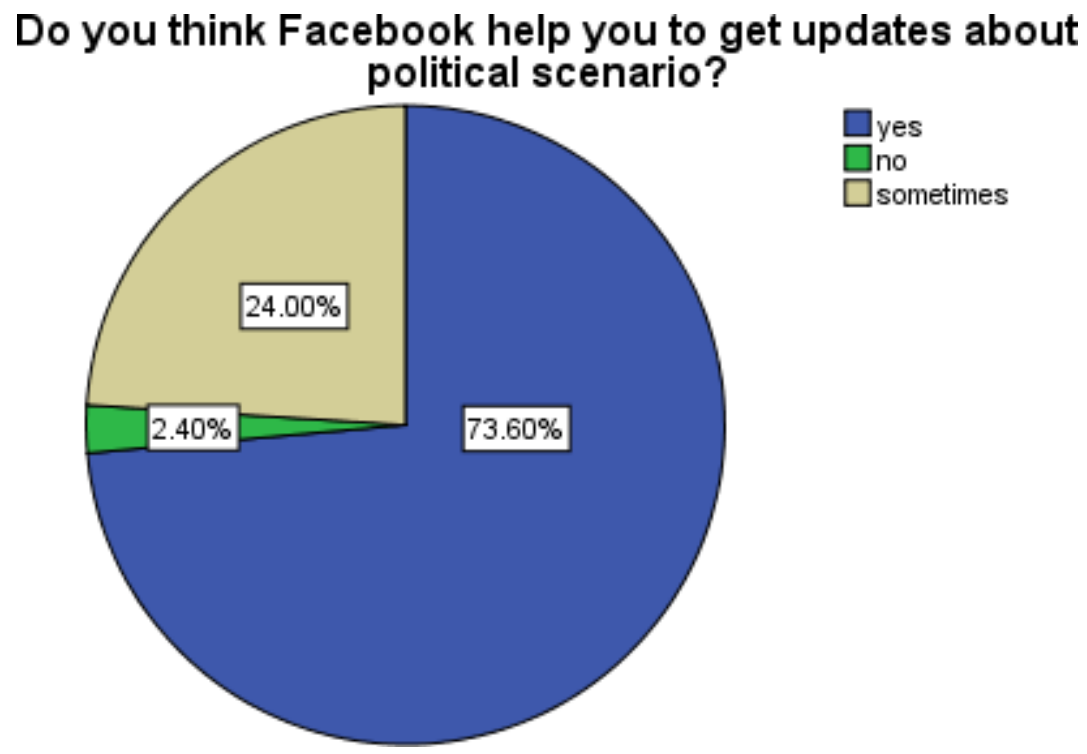

Table 1.12 shows Facebook helps youth to get update regarding politics

\begin{tabular}{|l|r|r|r|r|}
\hline & Frequency & Percent & Valid Percent & $\begin{array}{c}\text { Cumulative } \\
\text { Percent }\end{array}$ \\
\hline Yes & 184 & 73.6 & 73.6 & 73.6 \\
No & 6 & 2.4 & 2.4 & 76.0 \\
Sometimes & 60 & 24.0 & 24.0 & 100.0 \\
Never & 0 & .0 & .0 & 0.0 \\
Total & 250 & 100.0 & 100.0 & \\
\hline
\end{tabular}

Table 1.12 shows that Facebook help youth to get updates about political scenario to which $73.6 \%$ responded yes that Facebook updates them about political scenario whereas $2.4 \%$ answered no that Facebook do not update them about political scenario. $24 \%$ responded Sometimes it updates however, $0 \%$ answered as never.

Q13: Are you interested to follow Facebook posts that discuss politics? 
Figure 1.13 shows youth interest to follow political posts

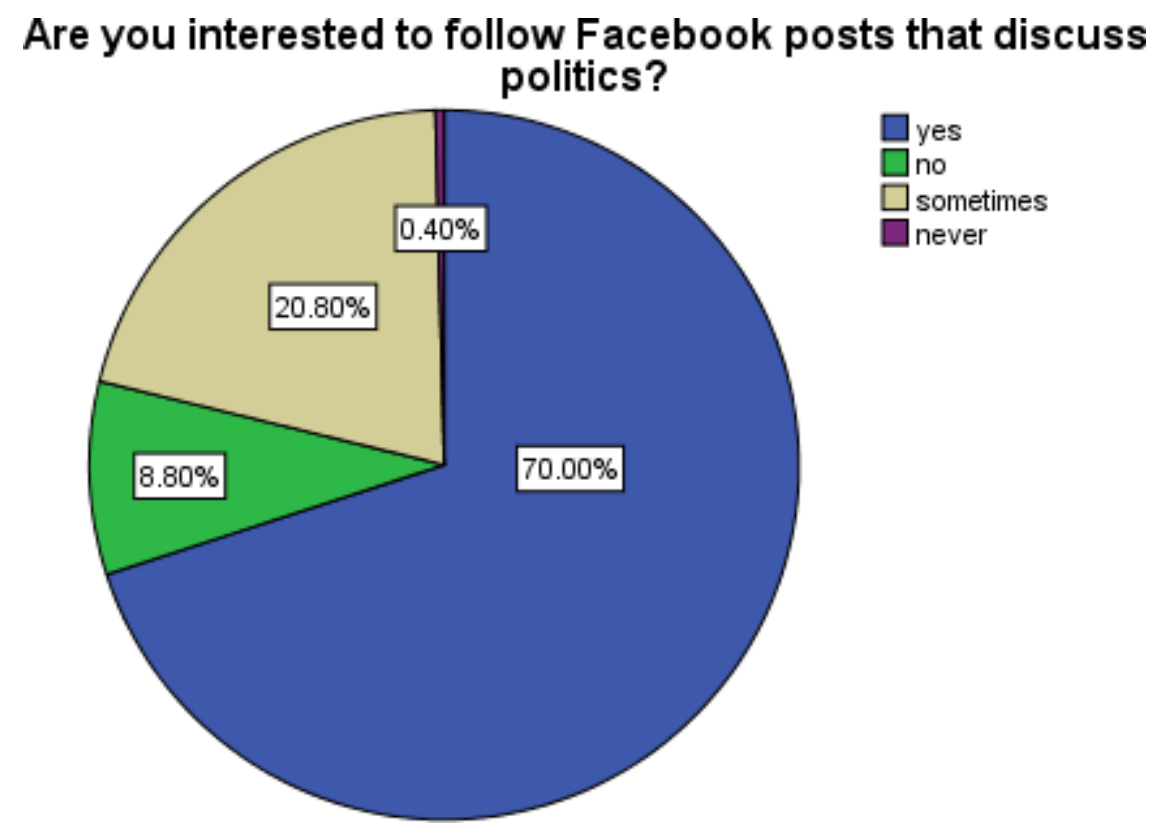

Table 1.13 shows youth interest to follow political posts

\begin{tabular}{|l|r|r|r|r|}
\hline & Frequency & Percent & Valid Percent & $\begin{array}{c}\text { Cumulative } \\
\text { Percent }\end{array}$ \\
\hline Yes & 175 & 70.0 & 70.0 & 70.0 \\
No & 22 & 8.8 & 8.8 & 78.8 \\
Sometimes & 52 & 20.8 & 20.8 & 99.6 \\
Never & 1 & .4 & .4 & 100.0 \\
Total & 250 & 100.0 & 100.0 & \\
\hline
\end{tabular}

Table 1.13 shows youth interest to follow Facebook posts which discuss politics to which $70 \%$ responded yes that they follow Facebook posts about politics, while $8.8 \%$ answered No that they do not follow Facebook posts which discuss politics, 20.8\% responded that sometimes they follow Facebook posts regarding politics however, $4 \%$ replied that they never follow Facebook posts that discuss politics.

Q14: Do you follow a political candidate page or group on Facebook? 
Figure 1.14 shows youth interest to follow a political candidate page or group

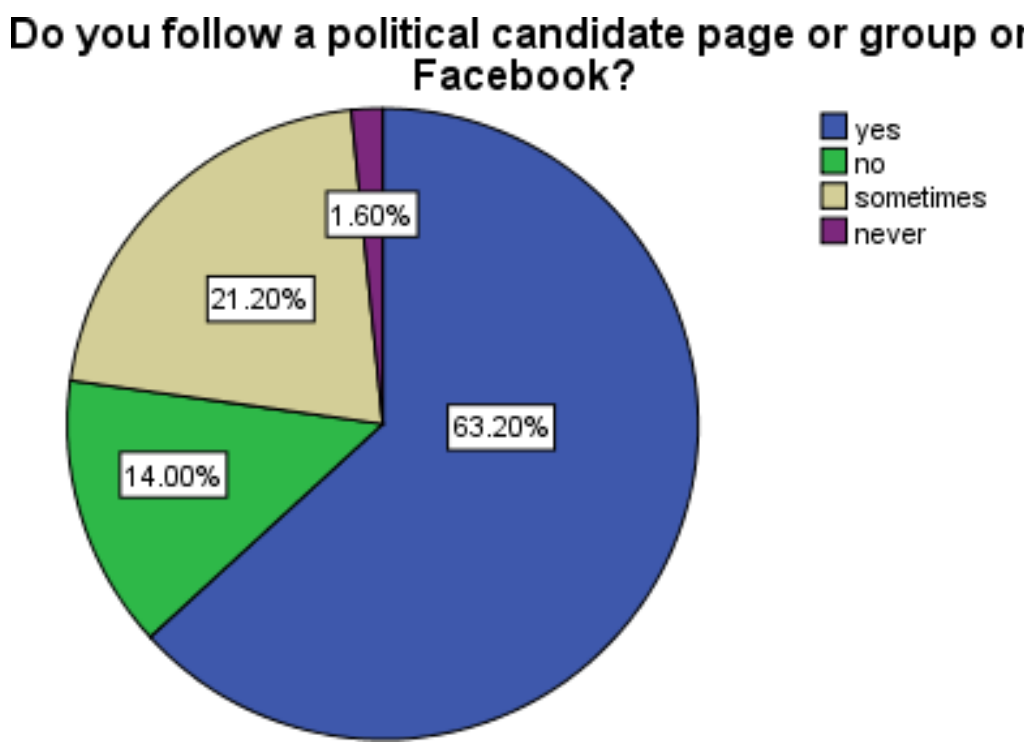

Table 1.14 shows youth interest to follow a political candidate page or group

\begin{tabular}{|l|r|r|r|r|}
\hline & Frequency & Percent & Valid Percent & \multicolumn{2}{c|}{$\begin{array}{c}\text { Cumulative } \\
\text { Percent }\end{array}$} \\
\hline Yes & 175 & 70.0 & 70.0 & 70.0 \\
No & 22 & 8.8 & 8.8 & 78.8 \\
Sometimes & 52 & 20.8 & 20.8 & 99.6 \\
Never & 1 & .4 & .4 & 100.0 \\
Total & 250 & 100.0 & 100.0 & \\
\hline
\end{tabular}

Table 1.14 shows youth interest to follow political candidate page or group on Facebook to which $63.2 \%$ replied yes that they follow these pages whereas $14 \%$ answered no that do not follows political candidate or group. $21.2 \%$ responded sometimes they follow these pages however, $1.6 \%$ replied never.

Q.15: Do you participate in Facebook political polls? 
Figure 1.15 shows participation in Facebook political posts

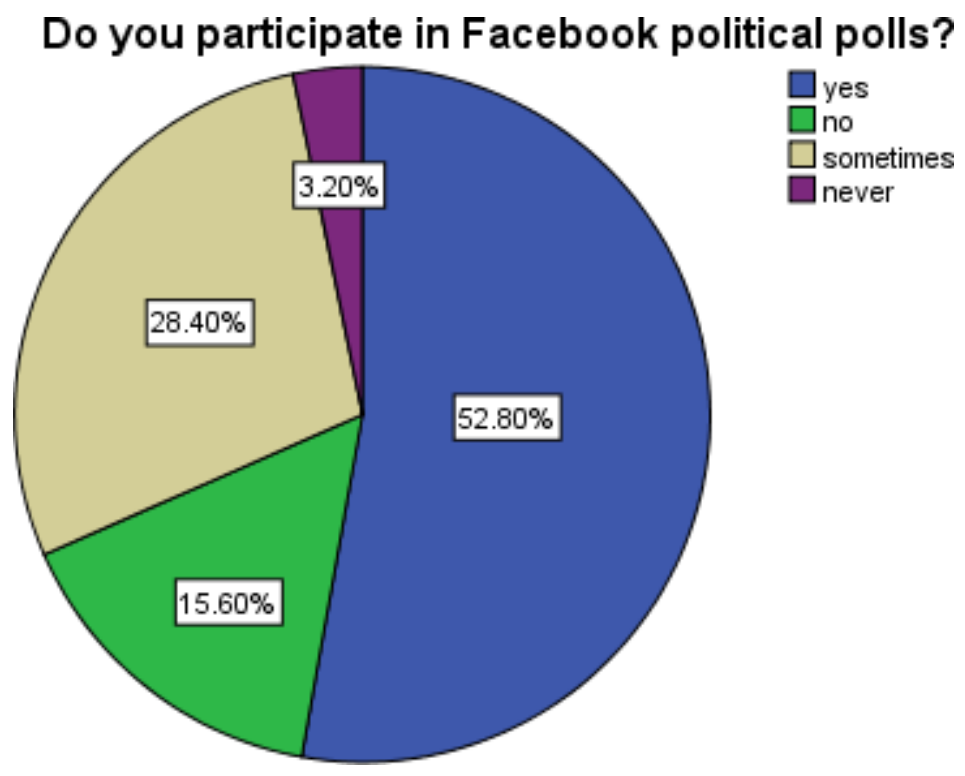

Table 1.15 shows participation in Facebook political posts

\begin{tabular}{|l|r|r|r|r|}
\hline & Frequency & Percent & Valid Percent & $\begin{array}{c}\text { Cumulative } \\
\text { Percent }\end{array}$ \\
\hline Yes & 132 & 52.8 & 52.8 & 52.8 \\
No & 39 & 15.6 & 15.6 & 68.4 \\
Sometimes & 71 & 28.4 & 28.4 & 96.8 \\
Never & 8 & 3.2 & 3.2 & 100.0 \\
Total & 250 & 100.0 & 100.0 & \\
\hline
\end{tabular}

Table 1.15 shows youth participation in political polls on Facebook to which $52.8 \%$ answered yes; they participate in political polls while $15.6 \%$ answered no; they do not follow, $28.4 \%$ replied sometimes and $3.2 \%$ said never.

Q16: Do you prefer to add your political information on Facebook profile? 
Figure 1.16 shows youth preference to add political information

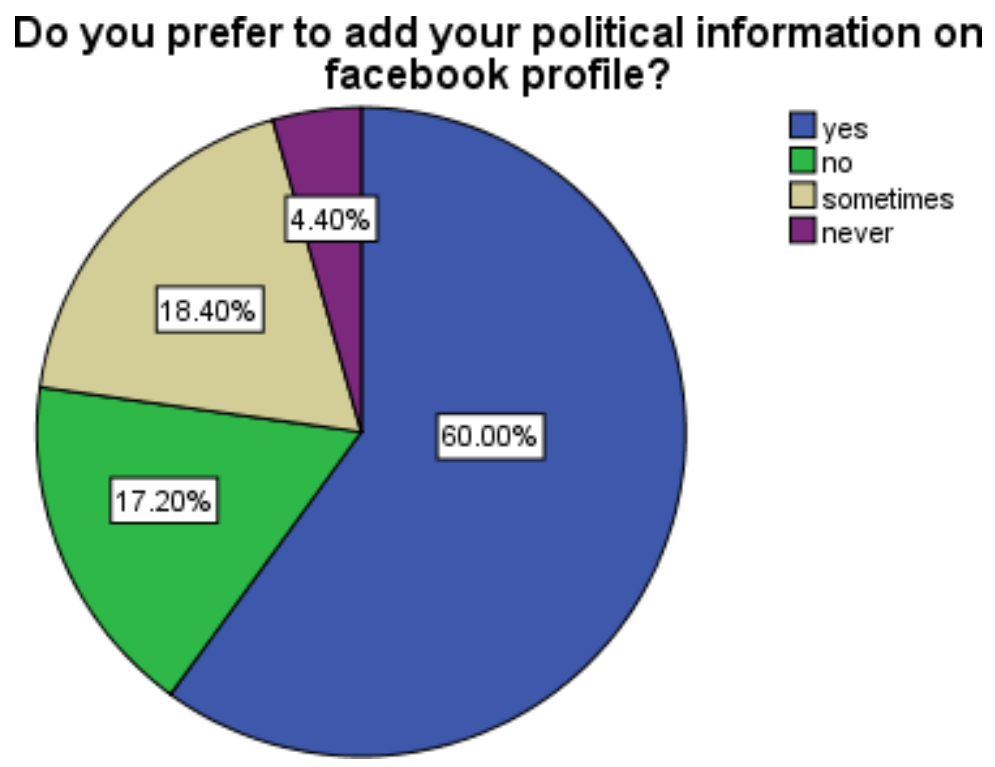

Table 1.16 shows youth preference to add political information

\begin{tabular}{|l|r|r|r|r|}
\hline & Frequency & Percent & Valid Percent & $\begin{array}{c}\text { Cumulative } \\
\text { Percent }\end{array}$ \\
\hline Yes & 150 & 60.0 & 60.0 & 60.0 \\
No & 43 & 17.2 & 17.2 & 77.2 \\
Sometimes & 46 & 18.4 & 18.4 & 95.6 \\
Never & 11 & 4.4 & 4.4 & 100.0 \\
Total & 250 & 100.0 & 100.0 & \\
\hline
\end{tabular}

Table 1.16 shows that youth prefer to add their political information on Facebook profile to which $60 \%$ answered yes; they add their political information on their profiles, $17.2 \%$ replied no while $18.4 \%$ answered sometimes they add their political information and $4.4 \%$ responded never.

Q. 17: Do you post status updates about politics on Facebook? 
Figure 1.17 shows status updates regarding politics

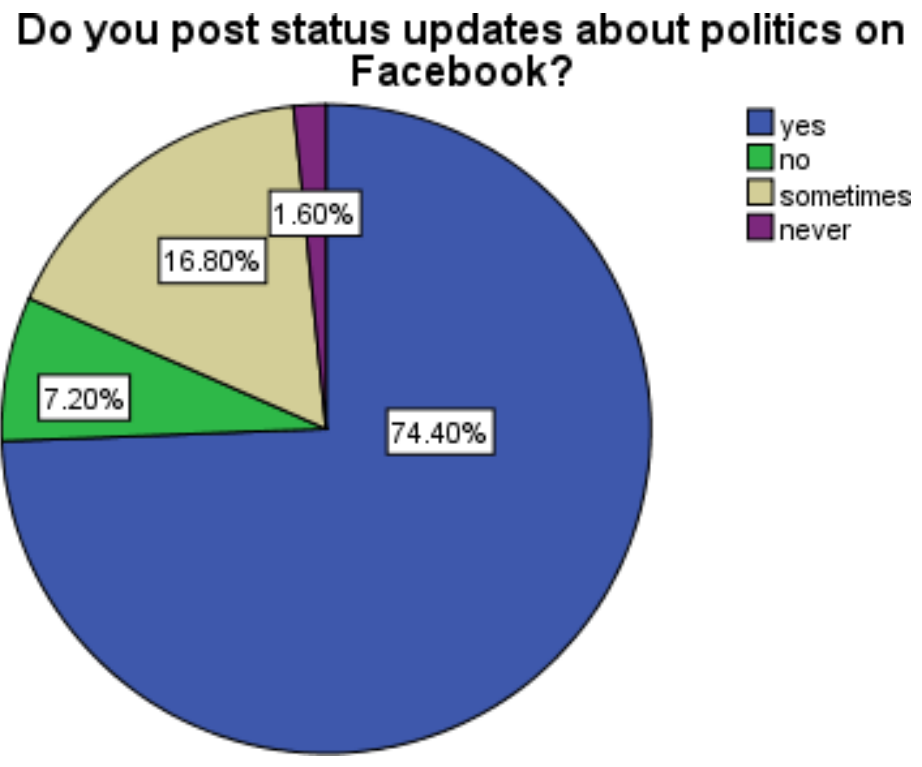

Table 1.17 shows status updates regarding politics

\begin{tabular}{|l|r|r|r|r|}
\hline & Frequency & Percent & Valid Percent & $\begin{array}{c}\text { Cumulative } \\
\text { Percent }\end{array}$ \\
\hline Yes & 186 & 74.4 & 74.4 & 74.4 \\
No & 18 & 7.2 & 7.2 & 81.6 \\
Sometimes & 42 & 16.8 & 16.8 & 98.4 \\
Never & 4 & 1.6 & 1.6 & 100.0 \\
Total & 250 & 100.0 & 100.0 & \\
\hline
\end{tabular}

Table 1.17 shows that youth post status updates on their profiles, $74.4 \%$ responded yes they post status regarding politics, $7.2 \%$ replied no they do not post statuses about politics. However $16.8 \%$ answered sometimes and $1.6 \%$ responded never.

Q18: Do you post photos related to politics on Facebook? 
Figure 1.18 shows post of political photos

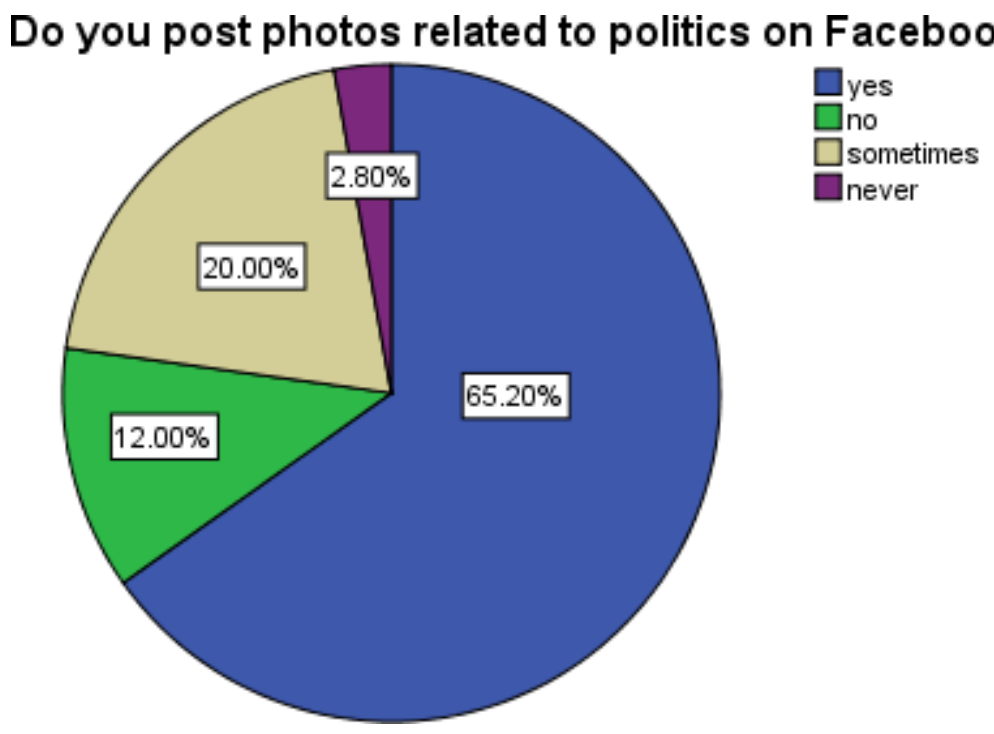

Table 1.18 shows post of political photos

\begin{tabular}{|l|r|r|r|r|}
\hline & Frequency & Percent & Valid Percent & $\begin{array}{c}\text { Cumulative } \\
\text { Percent }\end{array}$ \\
\hline Yes & 163 & 65.2 & 65.2 & 65.2 \\
No & 30 & 12.0 & 12.0 & 77.2 \\
Sometimes & 50 & 20.0 & 20.0 & 97.2 \\
Never & 7 & 2.8 & 2.8 & 100.0 \\
Total & 250 & 100.0 & 100.0 & \\
\hline
\end{tabular}

Table 1.18 shows that youth post photos related to politics on their profiles, $65.2 \%$ responded yes they post photos regarding politics, $12 \%$ replied as no they do not post photos about politics. However, $20 \%$ answered sometimes and $7 \%$ responded Never.

Q 19 : Do you post comments about politics on Facebook? 
Figure 1.19 shows post of comments about politics
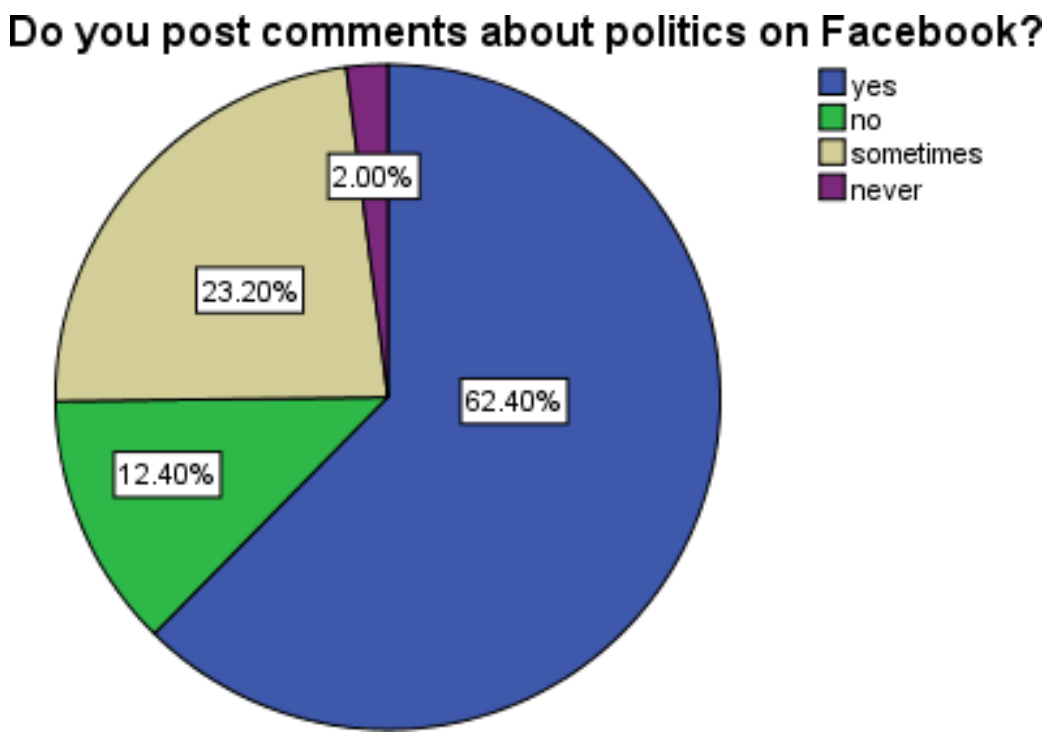

Table 1.19 shows post of comments about politics

\begin{tabular}{|l|r|r|r|r|}
\hline & Frequency & Percent & Valid Percent & $\begin{array}{c}\text { Cumulative } \\
\text { Percent }\end{array}$ \\
\hline Yes & 156 & 62.4 & 62.4 & 62.4 \\
No & 31 & 12.4 & 12.4 & 74.8 \\
Sometimes & 58 & 23.2 & 23.2 & 98.0 \\
Never & 5 & 2.0 & 2.0 & 100.0 \\
Total & 250 & 100.0 & 100.0 & \\
\hline
\end{tabular}

Table 1.19 shows that youth post comments about politics on Facebook, 62.4\% responded yes they post comments regarding politics, $12.4 \%$ replied no they do not post comments about politics whereas $23.3 \%$ answered sometimes and $2 \%$ replied Never.

Q 20: Do you post links about politics on Facebook? 
Figure 1.20 shows post of links about politics

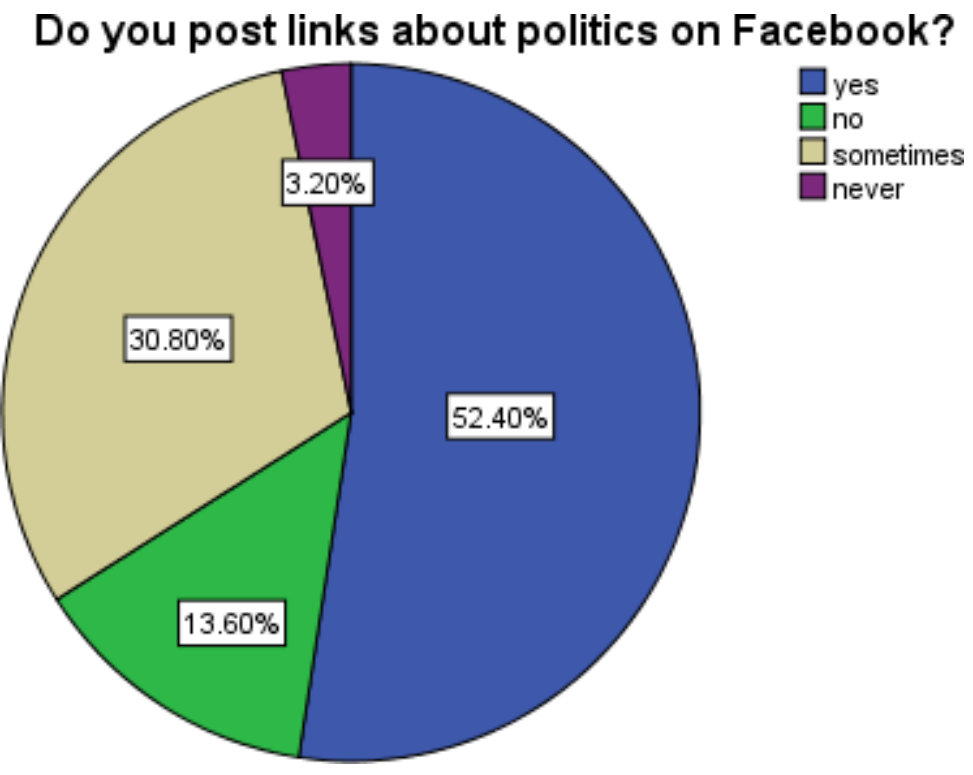

Table 1.20 shows post of links about politics

\begin{tabular}{|l|r|r|r|r|}
\hline & Frequency & Percent & Valid Percent & $\begin{array}{c}\text { Cumulative } \\
\text { Percent }\end{array}$ \\
\hline Yes & 131 & 52.4 & 52.4 & 52.4 \\
No & 34 & 13.6 & 13.6 & 66.0 \\
Sometimes & 77 & 30.8 & 30.8 & 96.8 \\
Never & 8 & 3.2 & 3.2 & 100.0 \\
Total & 250 & 100.0 & 100.0 & \\
\hline
\end{tabular}

Table 1.20 shows that youth post links about politics on their Facebook profiles to which 52.4\% responded as yes they post links regarding politics, $13.6 \%$ answered no they do not post links about politics. However, $30.8 \%$ answered sometimes and $3.2 \%$ responded Never.

Q. 21: Do you post political quotes or signs on Facebook? 
Figure 1.21 shows post of political quotes and signs

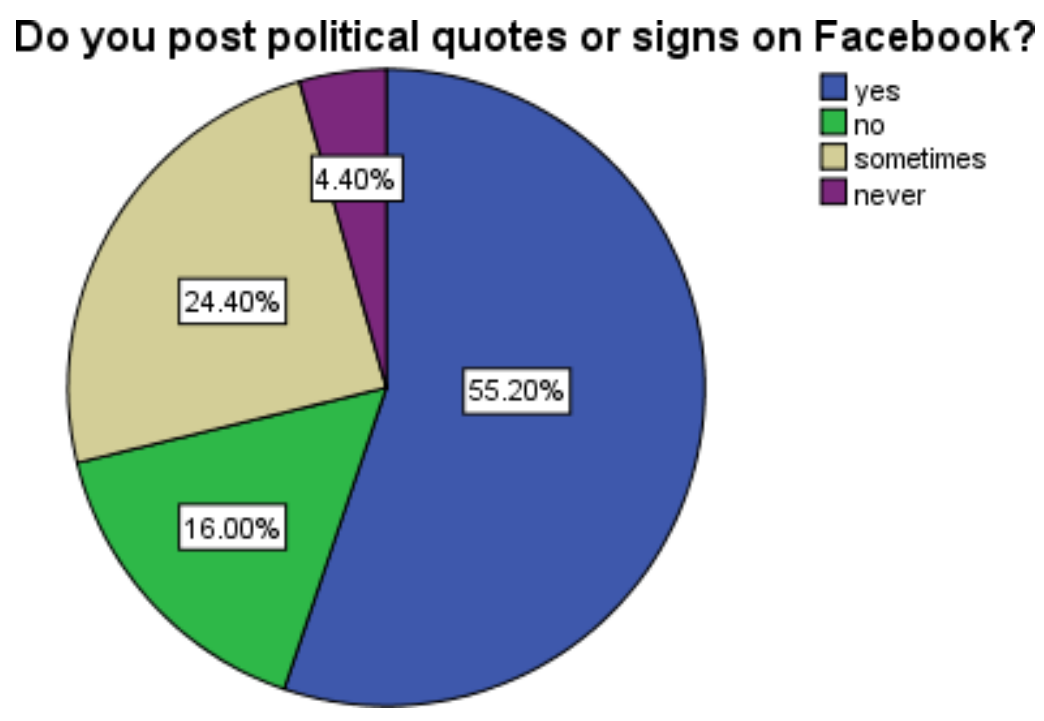

Table 1.21 shows post of political quotes and signs

\begin{tabular}{|c|r|r|r|r|}
\hline & Frequency & Percent & Valid Percent & $\begin{array}{c}\text { Cumulative } \\
\text { Percent }\end{array}$ \\
\hline Yes & 138 & 55.2 & 55.2 & 55.2 \\
No & 40 & 16.0 & 16.0 & 71.2 \\
Sometimes & 61 & 24.4 & 24.4 & 95.6 \\
Never & 11 & 4.4 & 4.4 & 100.0 \\
Total & 250 & 100.0 & 100.0 & \\
\hline
\end{tabular}

Table 1.21 shows that youth post political quotes or sign on their Facebook profiles, 55.2\% responded as yes they post comments politics signs and quotes, $16 \%$ replied as no they do not post political quotes and signs whereas $24.4 \%$ answered sometimes and $4.4 \%$ replied Never.

Q. 22: Do you respond to political invitations on Facebook? 
Figure 1.22 shows response to political invitations

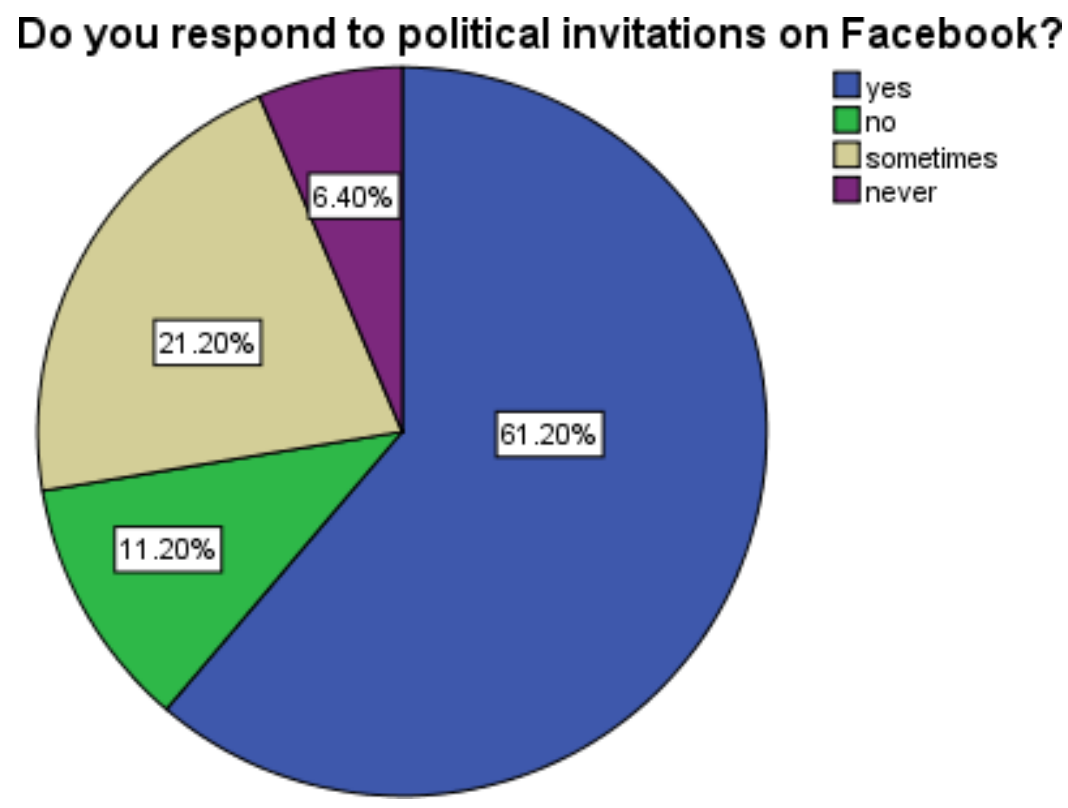

Table 1.22 shows response to political invitations

\begin{tabular}{|l|r|r|r|r|}
\hline & Frequency & Percent & Valid Percent & $\begin{array}{c}\text { Cumulative } \\
\text { Percent }\end{array}$ \\
\hline Yes & 153 & 61.2 & 61.2 & 61.2 \\
No & 28 & 11.2 & 11.2 & 72.4 \\
Sometimes & 53 & 21.2 & 21.2 & 93.6 \\
Never & 16 & 6.4 & 6.4 & 100.0 \\
Total & 250 & 100.0 & 100.0 & \\
\hline
\end{tabular}

Table 1.22 shows youth opinion towards responding to political invitations on Facebook, $61.2 \%$ answered yes; they respond political invitations while $11.2 \%$ responded no; they do not respond, $21.2 \%$ replied sometimes and $6.4 \%$ answered never.

\section{DISCUSSION AND ANALYSIS}

This research has been placed under the theory of Agenda setting and technological determinism theory. The agenda setting theory states that media plays a strong role in shaping the public opinion. As media prioritize issues and emphasize on particular subjects media transforms 
and edits the issue in order to presents it to the audiences. Therefore, media generate agenda for public and in turn audience considers the issue as more important. In technological determinism theory, it is stated that new media have changed the behavior patterns of people and altered the way of thinking which is because of advancement in technology. New technologies are changing the societies and it is bringing change in every individuals lives. In this perspective, the mode of social media acts as a platform in agenda formation on political issues. In this context, face book is one of the most extensively used social media platform within the masses.

On asking question about Facebook encourages youth to participate in politics, $74.8 \%$ of the respondents answered yes that Facebook encourages them to take part in politics, $4.4 \%$ responded that Facebook do not encourage them to participate in politics. However, $20.8 \%$ claimed that sometimes Facebook encourage them and $0 \%$ responded Never.

The question related to popularity of Facebook among youth for political participation explicates the widespread use of Facebook. It was found that a higher degree of respondents $72.8 \%$ responded that Facebook is popular among them for participating in politics. Whereas, 23. 6\% answered sometimes they use Facebook. On the other hand, only $2.8 \%$ replied that they do not use Facebook for political participation.

The results further illustrated that Facebook is considered as the main source of political news for youth. $56.4 \%$ of the total population answered in favored whereas $9.2 \%$ responded that Facebook is not main source of political news for them while $32.8 \%$ replied sometimes it is main source for getting political news. It is thus apparent that youth rely on Facebook for getting various kinds of political information.

Social media has gradually become a main source of expression opinions and thoughts. In this regard, 63.6\% of the total population responded that Facebook is the best platform to express your thoughts and opinions. In addition, upon asking question regarding youth spending most of their time on Facebook, $77.6 \%$ of the total respondents answered that they spend most of time on Facebook. However ,3.2\% answered that they do not spend most of their time on Facebook. Moreover, 19.2\% replied that they sometime spend their most of time on Facebook whereas 0\% replied responded that they never spend time on Facebook.

When asking that youth opinion about political affairs is influenced by Facebook activities, $68.4 \%$ of the total respondents answered that their opinion about political affairs is influenced by Facebook activities, $6.8 \%$ responded that their opinion is not influenced by activities on Facebook However $23.6 \%$ answered sometimes and $1.2 \%$ responded as never.

On inquiring that Facebook can be used as a political tool $66 \%$ responded that it can be used as a political tool whereas $6 \%$ of the total population answered that it cannot be used as a political tool. However, $26 \%$ of respondents replied sometimes it can be used as a political tool and $2 \%$ responded that Facebook can never be used as a political tool. This particular trend can be further examined by observing that how political leaders and political parties use 
Facebook accounts, groups and pages to connect with the society. Therefore, it is pertinent that Facebook acts as an influencer in shaping and reshaping opinion and perceptions. When inquiring youth about following a political candidate or group on Facebook, 63.2\% of the total respondents answered yes that they follow these pages whereas $14 \%$ responded no that do not follow political candidate or group pages while $21.2 \%$ said Sometimes they follow these pages and $1.6 \%$ of the total respondents answered Never.

The question regarding key role of Facebook in mobilizing youth to take part in politics, $66.8 \%$ of the total respondents answered that Facebook has played a key role to motivate youth for political participation, $6.8 \%$ replied that they do not think that Facebook mobilize them to take part in politics while $25.6 \%$ of the population answered Sometimes and $8 \%$ responded as never.

Upon asking that Facebook help youth to get updates about political scenario, $73.6 \%$ of the total population responded yes that Facebook updates them about political scenario whereas $2.4 \%$ answered that Facebook do not update them about political scenario. However, $24 \%$ responded sometimes it updates however, $0 \%$ said that Facebook never help to get updates about political scenario.

On asking question from the respondents about youth having interest to follow Facebook posts which discuss politics, $70 \%$ of the total population respondent yes that they follow Facebook posts about politics, while $8.8 \%$ answered that they do not follow Facebook posts which discuss politics. Although $20.8 \%$ said that sometimes they follow Facebook posts regarding politics however, $4 \%$ replied that they never follow Facebook posts that discuss politics.

Upon asking respondents regarding participation in political polls on Facebook, 52.8\% answered yes; they participate in political polls, $15.6 \%$ of the total respondents responded no; they do not take part in political polls on Facebook. However, 28.4\% answered sometimes and 3.2\% responded that they never take part in Facebook political tools.

\section{Conclusion}

Facebook helps youth to participate in the politics of Pakistan thus proving Hypothesis II. Facebook is capable of affecting the perceptions of youth. The primary spotlight was on Facebook and its utilization towards political support in which researchers came to realize that there is an effect on assessment development and reliance in youth with respect to political news and updates. Facebook is an extension that interfaces youth with political pioneers. The adolescent is currently more engaged with respect to taking part in legislative issues by utilizing Facebook. The results demonstrated that web-based media is going about as a political field in which youth can without much of a stretch get data, refreshes and takes an interest in governmental issues. Numerous respondents concurred that Facebook is making political mindfulness which assist them in making their political perspectives. Facebook is turning out to be new method of correspondence among youth since they think that it's appealing and benevolent. The crux of the matter is that long range informal communication destinations has no gatekeeping impact so it grants youth to take part in various exercises. The significant 
Pak. Journal of Int'L Affairs, Vol 4, Issue 1 (2021)

Facebook as an Instrument of Political ...

advantage of Facebook is that it has multiple impacts. In spite of precarious popularity based circumstances in Pakistan youth get an opportunity to voice their opinions. 


\section{References}

Adeney, K. (2013). Describing Democracy-The Case of Pakistans Election. http://www.globalpolicyjournal.com/blog/19/06/2013/describing-democracy\% $2 \% 80 \% 93$-case-pakistan\%E2\%80\%99s-elections

Ahmad, K. \& Sheikh, K. A. (2020). Social Media and Youth Participatory Politics: A Study of University Students. A Research Journal of South Asian Studies, 28(2), 353-360 .

Ahn, J. (2011). The Effect of Social Network Sites on Adolescents' Social and Academic Development: Current Theories and Controversies. Journal of the American Society for Information Science and Technology, 62(8), 1435-1445. http://onlinelibrary.wiley.com/doi/10.1002/asi.21540/full

Cowie, J. (2011). Can Internet Tame Governments? Yale Global Online. https://yaleglobal.yale.edu/content/can-internet-tame-governments-part-i

Grow, G \& Ward, J. (2013). The Role of Authenticity in Electoral Social Media Campaigns. First Monday. http://firstmonday.org/ojs/index.php/fm/article/view/4269

Hauer, T. (2017). Technological Determinism and New Media. International Journal of English, Literature and Social Sciences, 2(2).

Khawaja, M. A. (2013). Pakistan: Good People and Evil Politics. http://www.pkarticleshub.com/2013/05/11/pakistan-good-people-and-evil-politics/

Lee, N. (2009). Facebook Nation. California: Springer.

Marichal, J. (2013). Political Facebook Groups: Micro-Acticism and The Digital Front Stage. http://firstmonday.org/ojs/index.php/fm/article/view/4653/3800.

McCombs, M. E. \& Shaw, D. L. (1993). The Evolution of Agenda-Setting Research: TwentyFive Years in The Marketplace of Ideas. Journal of Communication. 43(2), 58-67.

Siddiqui, H. (2013). Youth and The Social media Landscape in Pakistan. www://cipe.org/blog/2013/08/13/youth-and-the-social-media-landscape-inpakistan/\#.U1QfWs6NliY

Tufekci, Z. \& Christopher, W. (2012). Social Media and The Decision to Participate in Political Protest: Observations From Tahrir Square. Journal of Communication, 62(2), 363-379. http://onlinelibrary.wiley.com/doi/10.1111/j.1460-2466.2012.01629.x/abstract.

Velasquez, A., \& LaRose, R. (2015). Youth collective activism through social media: The role of collective efficacy. New Media \& Society, 17(6), 899-918.

Warraich, H. (2011). Pakistan's Social Media Landscape. Foreign Policy. https://foreignpolicy.com/2011/03/18/pakistans-social-media-landscape/. 
Zain, N. R. M. (2014). Agenda Setting Theory. International Islamic University Malaysia. https://www.researchgate.net/publication/321698436_Agenda_Setting_Theory 
